\title{
Transplantation of hypoxia-preconditioned mesenchymal stem cells improves infarcted heart function via enhanced survival of implanted cells and angiogenesis
}

Xinyang Hu, MD, a,c Shan Ping Yu, MD, PhD, ${ }^{c, d}$ Jamie L. Fraser, BA, ${ }^{c}$ Zhongyang Lu, MD, PhD, ${ }^{c}$ Molly E. Ogle, BS, ${ }^{c}$ Jian-An Wang, MD, $\mathrm{PhD}^{\mathrm{b}}$ and Ling Wei, $\mathrm{MD}^{\mathrm{c}}$

From the Department of Cardiology, Sir Run Run Shaw Hospital, ${ }^{\mathrm{a}}$ Department of Cardiology, Second Affiliated Hospital, Zhejiang University, College of Medicine, Hangzhou, China, ${ }^{\mathrm{b}}$ Department of Pathology and Laboratory Medicine, ${ }^{\mathrm{c}}$ and Department of Pharmaceutical and Biomedical Sciences, Medical University of South Carolina, Charleston, SC.

This work was supported by National Institutes of Health grants NS 37372, NS 045155, and NS 045810 and American Heart Association and Bugher Foundation (AHA-Bugher) Awards 0170064N and $0170063 \mathrm{~N}$. The work was also supported by National Institutes of Health grant $\mathrm{C} 06$ RR015455 from the Extramural Research Facilities Program of the National Center for Research Resources.

Received for publication April 19, 2007; revisions received July 3, 2007; accepted for publication July 9, 2007.

Address for reprints: Ling Wei, MD, Department of Pathology and Laboratory Medicine, 165 Ashley Ave, Medical University of South Carolina, Charleston, SC 29425 (E-mail: weil@musc.edu).

J Thorac Cardiovasc Surg 2008;135:799808

$0022-5223 / \$ 34.00$

Copyright $(\odot 2008$ by The American Association for Thoracic Surgery

doi:10.1016/j.jtcvs.2007.07.071
Objectives: This study explored the novel strategy of hypoxic preconditioning of bone marrow mesenchymal stem cells before transplantation into the infarcted heart to promote their survival and therapeutic potential of mesenchymal stem cell transplantation after myocardial ischemia.

Methods: Mesenchymal stem cells from green fluorescent protein transgenic mice were cultured under normoxic or hypoxic ( $0.5 \%$ oxygen for 24 hours) conditions. Expression of growth factors and anti-apoptotic genes were examined by immunoblot. Normoxic or hypoxic stem cells were intramyocardially injected into the peri-infarct region of rats 30 minutes after permanent myocaridal infarction. Death of mesenchymal stem cells was assessed in vitro and in vivo after transplantation. Angiogenesis, infarct size, and heart function were measured 6 weeks after transplantation.

Results: Hypoxic preconditioning increased expression of pro-survival and proangiogenic factors including hypoxia-inducible factor 1, angiopoietin-1, vascular endothelial growth factor and its receptor, Flk-1, erythropoietin, Bcl-2, and Bcl-xL. Cell death of hypoxic stem cells and caspase- 3 activation in these cells were significantly lower compared with that in normoxic stem cells both in vitro and in vivo. Transplantation of hypoxic versus normoxic mesenchymal stem cells after myocardial infarctiion resulted in an increase in angiogenesis, as well as enhanced morphologic and functional benefits of stem cell therapy.

Conclusions: Hypoxic preconditioning enhances the capacity of mesenchymal stem cells to repair infarcted myocardium, attributable to reduced cell death and apoptosis of implanted cells, increased angiogenesis/vascularization, and paracrine effects.

$\mathrm{M}$ yocardial infarction (MI) induces the irreversible loss of cardiomyocytes, scar formation, and may ultimately result in congestive heart failure. ${ }^{1}$ Bone marrow mesenchymal stem cells (MSCs) are multipotent adult stem cells ${ }^{2}$ and able to differentiate into endothelial cells, vascular smooth muscle cells, and perhaps even cardiac-like myocytes when transplanted into the ischemic heart. ${ }^{3}$ Both animal and clinical studies have provided evidence that MSC transplantation can improve cardiac function through possible myogenesis and angiogenesis after MI. ${ }^{4,5}$ Some studies, however, have failed to observe the therapeutic effects of MSC transplantation ${ }^{6,7}$; thus, there is a need for further investigation into the use of MSCs and the improvement of transplantation techniques after MI.

A major dilemma in stem cell therapy for ischemic heart diseases is the low survival of transplanted cells in the ischemic and peri-infarcted region. ${ }^{6,8}$ Most implanted cells may die within 4 days after transplantation into the ischemic heart. ${ }^{9}$ Endogenous and environmental factors, such as the inflammatory response, may contribute to cell death. Thus, improving grafted cell survival after transplantation is critical for enhancing the efficacy and efficiency of stem cell therapy. 


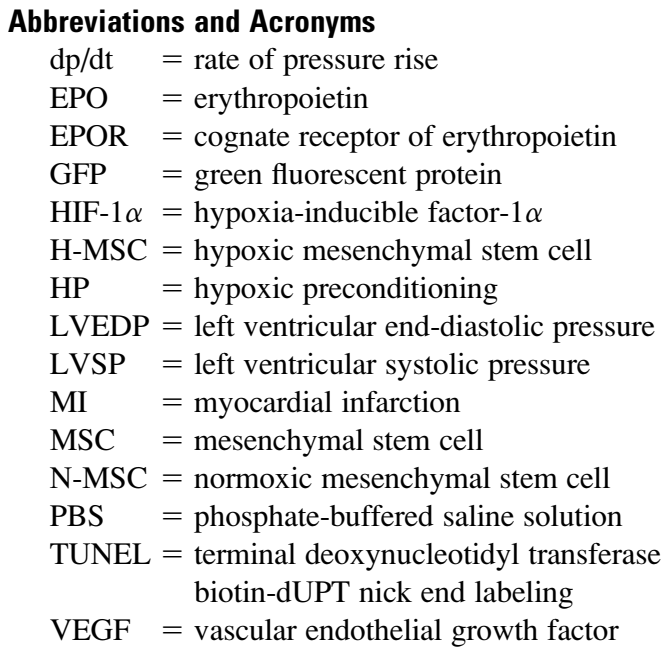

Angiogenesis remains one of the putative mechanisms in cardiac functional recovery after MI and stem cell transplantation. ${ }^{10}$ Stimulating angiogenesis showed therapeutic effects in ischemic heart disease. ${ }^{11}$ Transplanted MSCs can stimulate angiogenesis after MI by secreting multiple angiogenic cytokines and differentiating into endothelial cells. ${ }^{12}$ It can be reasoned that enhancing the MSC's ability to promote angiogenesis will further increase the therapeutic potential of MSC transplantation after MI.

Hypoxic preconditioning (HP) by sublethal hypoxic insult stimulates endogenous mechanisms resulting in multiple responses including protein expressions that protect against future lethal hypoxia and other insults. HP can decrease apoptosis of neurons through induction of hypoxia-inducible factor- $1 \alpha$ $(\mathrm{HIF}-1 \alpha)^{13}$ and protect myocytes from hypoxia and reperfusion injury. ${ }^{14} \mathrm{HP}$ stimulates myocardial angiogenesis to an extent sufficient to exert significant cardioprotection after MI. ${ }^{15}$

Cell transplantation therapy and HP have been studied as separate research topics. $\mathrm{We}^{16}$ recently demonstrated that in vitro HP of embryonic stem cells significantly increased their survival and tissue repair capabilities after transplantation into the ischemic brain. On the basis of the well-documented manifold benefits of HP, we examined the hypothesis that HP of cultured MSCs would promote their survival in vitro as well as after transplantation. Furthermore, enhanced survival and trophic support would contribute to endothelial differentiation and stimulate angiogenesis. The HP strategy for stem cell transplantation would ultimately benefit functional recovery after MI.

\section{Materials and Methods MSC Culture}

Bone marrow MSCs were isolated and harvested as previously described. ${ }^{17}$ In brief, MSCs were acquired from the femoral and tibial bones of green fluorescent protein (GFP) transgenic mice. MSCs were flushed from the femurs and tibias of GFP mice using a 25guage needle. Mononuclear cells were suspended in Dulbecco's modified Eagle's medium supplemented with $10 \%$ fetal bovine serum and plated in flasks. Cultures were maintained at $37^{\circ} \mathrm{C}$ in a humidified atmosphere containing 5\% carbon dioxide. After 24 hours, nonadherent cells were discarded, and adherent cells were washed three times with phosphate-buffered saline solution (PBS). Fresh complete medium was added and replaced every 4 days. Each primary culture was subcultured 1:2 when MSCs grew to approximately $80 \%$ confluence. To confirm the cellular identity of cultured cells, we subjected MSCs to fluorescence-activated cell sorting using CD90, CD34, and CD45 markers, and cultured cells were identified as CD90+ and CD34-/CD45- cells.

\section{Sublethal Hypoxia Protocol}

For HP treatment, cells were subcultured 1:2 and cultured for 3 days until confluent. Fresh complete medium was added before hypoxia. Hypoxia treatment was achieved with a well-characterized, finely controlled ProOx-C-chamber system (Biospherix, Redfield, NY) for 24 hours. The oxygen concentration in the chamber was maintained at $0.5 \%$, with a residual gas mixture composed of $5 \%$ carbon dioxide and balanced nitrogen.

\section{Cell Death Assays in Vitro}

Cell death was assayed using trypan blue staining as described previously. ${ }^{18}$

\section{Western Blot Analysis}

Immunoblot was applied to determine the effect of HP on the expression of growth factors and antiapoptotic genes in MSCs. Cultured cells were lysed with modified radioimmunoprecipitation assay buffer (50 mmol/L HEPES, pH 7.3, 1\% sodium deoxycholate, $1 \%$ Triton $\mathrm{X}-100,0.1 \%$ sodium dodecylsulfate, $150 \mathrm{mmol} / \mathrm{L} \mathrm{NaCl}, 1$ $\mathrm{mmol} / \mathrm{L}$ ethylenediaminetetraacetic acid, $1 \mathrm{mmol} / \mathrm{L} \mathrm{Na}_{3} \mathrm{VO}_{4}, 1 \mathrm{mmol} /$ $\mathrm{L} \mathrm{NaF}$, and protease inhibitor cocktail [Roche, Nutley, NJ]) for 30 minutes, followed by centrifugation at $14,000 \mathrm{~g}$ for 30 minutes. Protein concentration of each sample was determined with the Bicinchoninic Acid Assay (Sigma Chemical Co, St Louis, Mo). Then, $40 \mu \mathrm{g}$ of protein per sample was electrophoresed on a $6 \%$ - to $15 \%$-gradient gel by sodium dodecylsulfate-polyacrylamide gel electrophoresis in a Hoefer Mini-Gel system (Amersham Biosciences, Piscataway, NJ) and transferred in a Hoefer Transfer Tank (Amersham Biosciences) to polyvinylidene difluoride membrane (Bio-Rad, Hercules, Calif). Membranes were blocked with $7 \%$ milk in Tris-buffered saline and $0.2 \%$ Tween at room temperature for 2 hours and were then incubated overnight at $4{ }^{\circ} \mathrm{C}$ with specific primary antibodies. The blots were washed three times with Tris-buffered saline and $0.2 \%$ Tween and incubated with alkaline phosphatase-conjugated secondary antibodies for 2 hours at room temperature. The expression signals were detected with 5-bromo-4-chloro-3-indolyl phosphate/nitroblue tetrazolium (BCIP/NBT) solution (Sigma).

\section{Rat Model of MI}

Male Wistar rats (280-330 g) were intubated under general anesthesia with $4 \%$ chloral hydrate $(4 \mathrm{mg} / \mathrm{kg}$, administered intraperitoneally) and ventilated with room air by a small animal ventilator (Vetronics, Lafayette, Ind). MI was induced by permanent ligation of the left anterior descending coronary artery with a 6-0 silk 
suture. ${ }^{19}$ Successful performance of coronary occlusion was verified by blanching of the myocardium distal to the coronary ligation. The sham-operation group received the same procedure of thoracotomy without coronary ligation. A minimum of 6 animals was in each experimental group. In our investigation, the MI-induced mortality rate was $\sim 15 \%$. We did not notice a significant difference between groups. The investigation conforms to the "Guide for the Care and Use of Laboratory Animals" published by the US National Institutes of Health (NIH Publication No. 85-23, revised 1996).

\section{Cell Preparation and Transplantation}

MSCs were labeled with Hoechst 33342 to track and count the implanted cells. In brief, sterile Hoechst 33342 was added to culture medium with a final concentration of $10 \mu \mathrm{mol} / \mathrm{mL}$ for 2 hours before transplantation. Then the cells were rinsed 6 times with PBS to remove unbound Hoechst 33342, detached with $0.25 \%$ (w/v) trypsin ethylenediaminetetraacetic acid, and suspended in complete medium. Cells were centrifuged and washed with PBS, followed by the final centrifugation and suspension in serum-free medium at $1 \times 10^{6}$ cells per $150 \mu \mathrm{L}$. Before transplantation, MSCs in the HP group (H-MSCs) were treated for 24 hours with sublethal hypoxia $(0.5 \%$ oxygen) and then reoxygenated in $20 \%$ oxygen for 2 hours. Half an hour after ligation of the left anterior descending coronary artery, $150 \mu \mathrm{L}$ of medium containing $1 \times 10^{6}$ cells was directly injected into the ischemic peri-infarct region. MI control rats received MI alone and received the same volume of serum free/cell free medium injection. Experimental groups were divided into four groups of 10 rats each: (1) MI with transplant of normoxic MSCs (N-MSCs), (2) MI with transplant of hypoxia-pretreated MSCs (H-MSCs), (3) MI with injection of control medium, and (4) sham-operated control group.

\section{Immunofluorescence Staining}

For immunofluorescence staining, cells on dishes and in heart tissues were fixed with $10 \%$ formalin for 10 minutes, permeabilized with $0.2 \%$ Triton X-100 for 5 minutes, and blocked with $1 \%$ fish gelatin (Sigma) for 1 hour at room temperature. Specimens were then incubated with primary antibodies overnight at $4^{\circ} \mathrm{C}$, then washed with PBS three times, and incubated with Cy3-conjugated donkey anti-rabbit immunoglobulin G (1:500; Jackson ImmunoResearsh, West Grove, Pa) or Alexa Fluor 488 anti-goat immunoglobulin G (1:200, Molecular Probes, Carlsbad, Calif) for 1 hour at room temperature. The cells were treated with Hoechst 33342 (1:20000; Molecular Probes, Carlsbad, Calif) for 5 minutes. Dishes or slides were mounted and analyzed under a florescent microscope (BX51, Olympus, Tokyo, Japan).

\section{Measurement of Hemodynamics}

Cardiac hemodynamics was measured at 6 weeks after myocardial ischemia. Rats were anesthetized with $4 \%$ chloral hydrate $(40 \mathrm{mg}$ / $\mathrm{kg}$, intraperitoneally). The carotid artery was isolated and cannulated with a microtip catheter that was connected with an MLT0699 disposable pressure transducer (ADInstrument, Colorado Springs, Colo). Left ventricular systolic pressure (LVSP), left ventricular end-diastolic pressure (LVEDP), maximum dp/dt $(+\mathrm{dp} / \mathrm{dt}$, pressure rise rate) and minimum $\mathrm{dp} / \mathrm{dt}(-\mathrm{dp} / \mathrm{dt}$, pressure decrease rate), and heart rate were monitored and recorded by the Powerlab/800 data acquisition system (ADInstrument).

\section{Infarct Size Measurement}

After measurement of hemodynamics, the animals were humanely sacrificed and the hearts were quickly harvested and divided into three transverse sections. Tissues from the free wall of the left ventricle including infarct and peri-infarct regions were then embedded in optimal cutting temperature (OCT) compound (Sakura Finetek USA Inc, Torrance, Calif). Frozen sections of left ventricular samples were cut at $10-\mu \mathrm{m}$ thickness and prepared for staining. To determine infarct size, hearts at papillary muscle level were selected and Masson trichrome and hematoxylin-eosin staining were performed. Images were digitized by the NIH image analysis system (National Institutes of Health, Bethesda, Md). Infarct size was calculated by dividing the sum of the planimetered endocardial and epicardial circumferences of the infarcted area by the sum of the total epicardial and endocardial circumferences of the left ventricle. ${ }^{20}$

\section{Terminal Deoxynucleotidyl Transferase Biotin-dUPT Nick End Labeling (TUNEL) in heart sections}

A TUNEL staining kit (DeadEnd Fluorometric TUNEL system, Promega, Madison, Wis) was used to visualize cell death in heart sections. After 10 minutes of fixing by $10 \%$ buffered formalin phosphate (Fisher Scientific, Pittsburgh, Pa) and pretreatment with $-20^{\circ} \mathrm{C}$ ethanol/acetic acid $(2: 1)$ and $0.2 \%$ Triton $\mathrm{X}-100$, the heart sections were incubated in an equilibration buffer as instructed by the kit. The TdT enzyme and nucleotide mix was then added at proportions specified by the kit for 75 minutes at room temperature. The slides were washed with $2 \times$ standard saline citrate washing buffer for 15 minutes and followed by three washings with PBS.

\section{Cell and Vessel Counting}

Cell count was performed using a design-based stereologic method. For counting caspase- 3 positive cells in cultures, 6 fields per dish were randomly chosen under $20 \times$ magnification of a fluorescent microscope $(n=4)$. For counting caspase-3, Hoechst 33342, and GFP-positive cells and vessels in heart sections, every tenth heart section $(100 \mu \mathrm{m}$ apart) across the entire region of interest was counted, and 6 fields per heart section were randomly chosen and photographed under $40 \times$ magnification with a fluorescent microscope; this was repeated in 4 separate sections per heart.

\section{Statistical Analysis}

The Student two-tailed $t$ test was used for comparison of two experimental groups. Multiple comparisons were done by 1-way analysis of variance followed by the Tukey post hoc test for multiple pairwise examinations. Data are expressed as the mean \pm standard error of mean.

\section{Results}

Effect of HP on Expression of Pro-survival and Angiogenic Factors

To characterize the effect of hypoxia on MSCs, we first analyzed the protein expression levels of growth and angiogenic factors in N-MSCs and H-MSCs by Western blotting. N-MSCs displayed endogenous expression of growth and angiogenic factors including HIF- $1 \alpha$, vascular endothelial growth factor (VEGF) and its receptor (Flk-1), angiopoietin-1, and erythropoietin (EPO) and its cognate receptor 
(EPOR). As expected, sublethal hypoxia significantly increased the HIF- $1 \alpha$ protein expression level (3.4-fold) as well as Flk-1, angiopoietin-1, EPO, and EPOR in H-MSCs (Figure 1).

Consistent with a model of HP as protective to cell survival, H-MSCs displayed a significant increase in the expression of pro-survival proteins p105, the nuclear factor $-\kappa \mathrm{B}$ subunits P65 and P50, and anti-apoptotic proteins Bcl-2 and Bcl-xL. Increased nuclear fractions of P65 and P50 further suggested activation and translocation of these two nuclear factor- $\kappa \mathrm{B}$ transcription factors (Figure 2). The hypoxia treated cells also had a significantly lower level of active caspase-3 than did N-MSCs (Figure 2).

\section{HP Promotes Cell Survival In Vitro and After Transplantation}

To determine the vulnerability of N-MSCs and H-MSCs to apoptosis, we subjected cells in culture to 24 hours of normoxia or sublethal hypoxia followed by 24 hours of serum

\section{A}

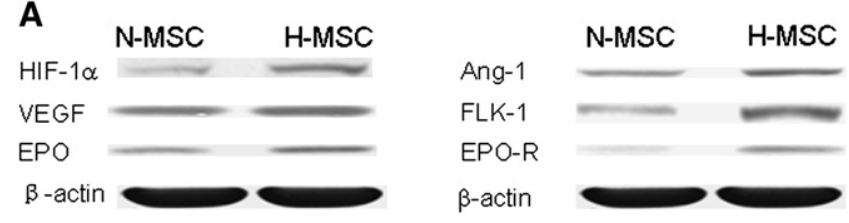

B

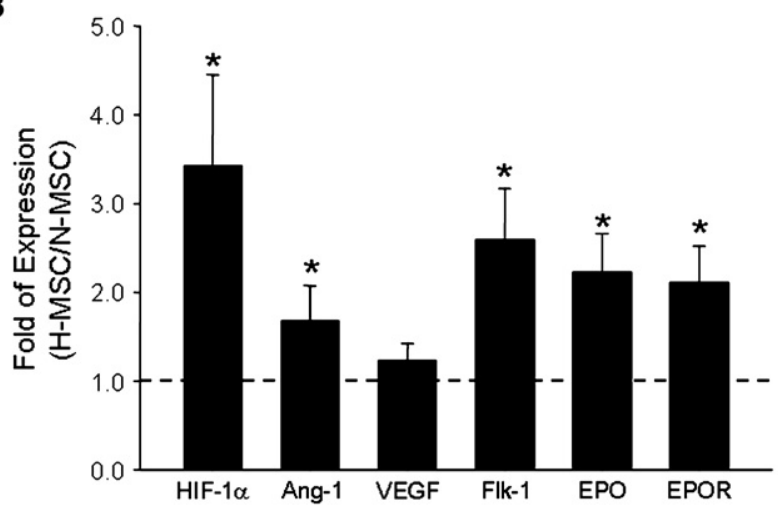

Figure 1. Hypoxic preconditioning up-regulated HIF-1 $\alpha$ and growth factor expression. HIF-1 $\alpha$ as well as pro-survival and pro-angiogenic factors Ang-1, VEGF, Flk-1, EPO, and EPOR were detected by Western blot. A, The expression levels of HIF-1 $\alpha$, Ang-1, VEGF, Flk-1, EPO, and the EPOR in N-MSCs and H-MSCs. Mouse $\beta$-actin was used as the loading control. B, Densitometry analysis for comparisons of the relative expression levels of different factors in H-MSCs with respect to N-MSCs (dotted line). $\mathbf{N}=6 .{ }^{*} \boldsymbol{P}<.05$ compared with $\mathbf{N}$-MSCs by the Student 2-tailed $t$ test. HIF-1 $\alpha$, Hypoxia-inducible factor-1 $\alpha ; N$-MSC, Normoxic mesenchymal stem cell; H-MSC; hypoxic mesenchymal stem cell; Ang-1, angiopoietin-1; VEGF, vascular endothelial growth factor; EPO, erythropoietin; EPOR, cognate receptor of erythropoietin. deprivation. Cells were then stained with trypan blue and caspase- 3 to assess cell death and apoptosis, respectively. The hypoxia treatment was confirmed to be sublethal with no significant increases in trypan blue (Figure 3 ) or caspase-3 staining (data not shown). Serum deprivation increased the ratio of trypan blue and caspase-3 staining positive cells in both $\mathrm{N}-\mathrm{MSCs}$ and H-MSCs; however, the increases were much less in H-MSCs than in N-MSCs (Figure 3). The percentage of trypan blue positive cells versus total cells was $24.3 \% \pm$ $5.4 \%$ and $39.3 \% \pm 6.8 \%$ in H-MSCs and N-MSCs, respectively ( $\mathrm{n}=4, P<.05$ ), and the percentage of caspase- 3 positive cells was $25.6 \% \pm 5.4 \%$ and $36.6 \% \pm 6.6 \%$, respectively (n $=4, P<.05)$ (Figure 3 ).

To assess whether HP might protect MSCs in vivo, we transplanted N-MSCs and H-MSCs into the peri-infarcted region 30 minutes after MI. Twenty-four hours later, the animals were humanely sacrificed and apoptosis of implanted cells was identified by the ratio of caspase-3/Hoechst/GFP co-labeled cells versus total transplanted cells pre-labeled with Hoechst and GFP; cell death was identified by the ratio of TUNEL/Hoechst positive cells versus total transplanted cells. In the H-MSC transplantation group, the implanted cell death and apoptosis-associated caspase-3 activation were significantly suppressed (Figure 4). To test whether HP might prevent delayed MSC cell death, we assessed cell death using TUNEL staining 72 hours after transplantation. In the H-MSC transplantation group, the ratio of TUNEL positive cells was significantly reduced (Figure 4).

\section{Enhanced Angiogenesis/Vascularization After MSC Transplantation}

To assess angiogenic and vascularization activity, we stained heart sections with the endothelial cell marker CD31 and arteriole marker smooth muscle actin. Six weeks after ischemia with or without MSC transplantation, both the total vessel density/area and arteriole density/area in both MSC transplantation groups were greater than those in the ischemiaonly group (Figure 5). Between the two transplantation groups, significantly greater total vessel density/area and arteriole density/area were observed in the H-MSC group than in the N-MSC group $(\mathrm{n}=6$ rats per group, $P<.05)$ (Figure 5). To further clarify whether HP promoted endothelial cell differentiation of MSCs, we calculated the ratio of CD31/Hoechst positive cells versus total transplanted cells pre-labeled with Hoechst. A higher endothelial cell differentiation ratio was identified in the $\mathrm{H}-\mathrm{MSC}$ transplantation group than in the N-MSC group $(\mathrm{n}=6, P<.05)$ (Figure 5$)$.

\section{Possible Myogenesis From Transplanted MSCs}

Myosin heavy chain staining showed that few MSCs expressed the myocyte protein myosin heavy chain in transplanted heart 6 weeks after transplantation (Figure 5). Our data, however, could not rule out the possibility that the co-labeling was due to cell fusion between exogenous and 
A

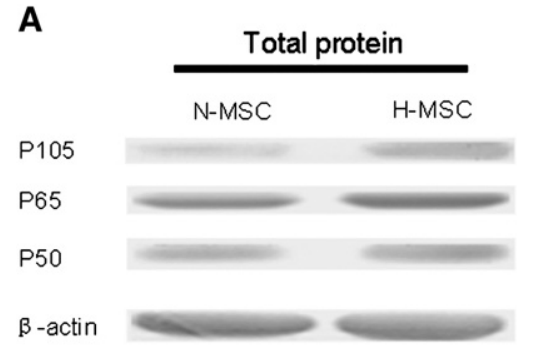

B

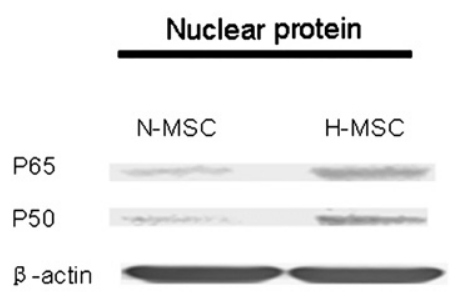

Figure 2. Effect of hypoxic preconditioning on anti-apoptotic gene expression. Western blot analysis of several key factors in cell survival, apoptosis and angiogenesis in N-MSCs and $\mathrm{H}$ MSCs. $A$ and $B$, Expression of NF- $K B$ precursor $P 105$, subunit $P 65$, and $P 50$ in cell lysate (A) and nuclear fraction (B). Mouse $\beta$-actin was used as the loading control. C, Densitometry analysis for comparisons of expression ratios of NF- $K$ B signals in H-MSCs with respect to the basal levels in N-MSCs (dotted line). D, Protein levels of Bcl2, Bcl-xL, and cleaved caspase-3 detected by Western blot. E, Densitometry of Bcl-2, Bcl-xL, and caspase-3 expression in H-MSCs compared with that in N-MSCs (dotted line). ${ }^{*} P<.05$ compared with N-MSCs by the Student 2-tailed $t$ test. For abbreviations, see Figure 1.

D

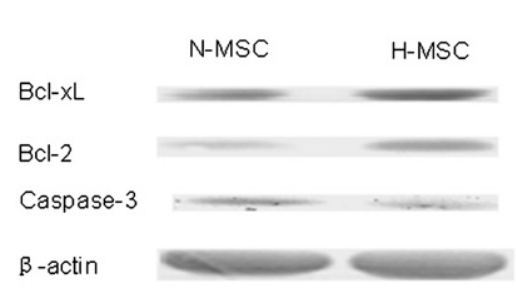

E

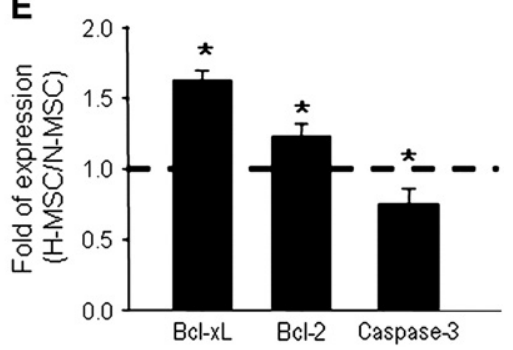

host cells. ${ }^{6,7}$ We also did not identify a noticeable effect of $\mathrm{HP}$ on this potential myogenesis.

\section{MSC Transplantation Reduces Infarct Size}

Hematoxylin-eosin staining and Masson trichrome staining of cardiac tissue revealed fibrosis in the infarct region 6 weeks after ischemia. Scar formation was evident in the ischemic hearts that received a medium control injection (Figure 6). The infarct size was reduced in both groups that received MSC transplantation; however, rats receiving H-MSC transplantation showed the smallest infarct size (Figure 6).

\section{Transplantation of MSCs improves cardiac function} Six weeks after ischemia, functional recovery of ischemic heart was measured by the LVSP, LVEDP, +dp/dt, and $-\mathrm{dp} / \mathrm{dt}$. MSC transplantation helped to prevent negative left ventricular remodeling. Rats that received N-MSCs had significantly increased LVSP and absolute values of $\pm \mathrm{dp} /$ $\mathrm{dt}$ compared with the control group (Figure 6). Even greater cardiac functional benefits were found in H-MSC transplan- tation group, showing higher LVSP, improved $\pm \mathrm{dp} / \mathrm{dt}$, and near normal LVEDP (Figure 6).

\section{Discussion}

The present study explored a novel preconditioning strategy to enhance the ability of MSCs to survive under pathologic conditions and promote their therapeutic effects within the ischemic heart. We have shown that sublethal hypoxia induces increases in several pro-survival and pro-angiogenic factors within the MSCs, contributing to the enhanced tolerance of $\mathrm{H}-\mathrm{MSCs}$ to apoptosis and increased angiogenesis after transplantation. Uemura and associates ${ }^{21}$ recently reported that transplantation of hypoxic preconditioned MSCs reduced the apoptosis of cardiomyocytes. In the present investigation, we provide new evidence that transplantation of hypoxic preconditioned MSCs promotes heart functional recovery through enhancing implanted cell survival and angiogenesis after MI.

Previous studies have shown that allogeneic MSCs are immunoregulatory and do not induce immune response in vitro $^{22}$ and in vivo ${ }^{23}$; xenogeneic bone marrow stromal 
Control
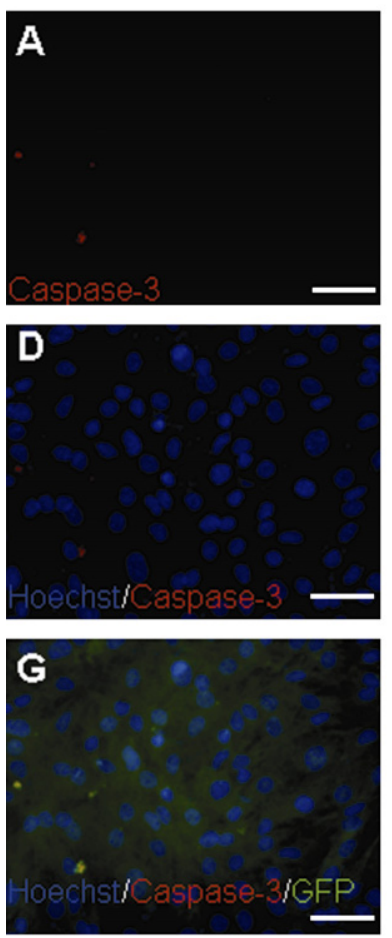

J

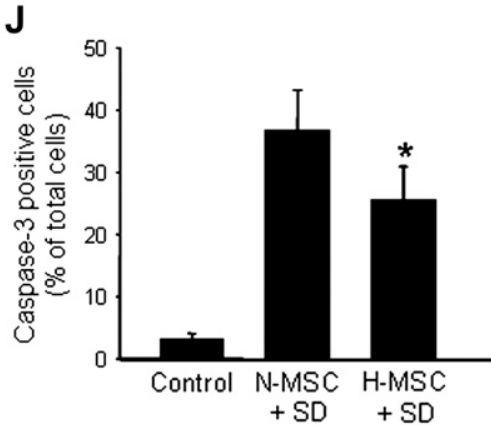

$\mathrm{N}-\mathrm{MSC}+\mathrm{SD}$
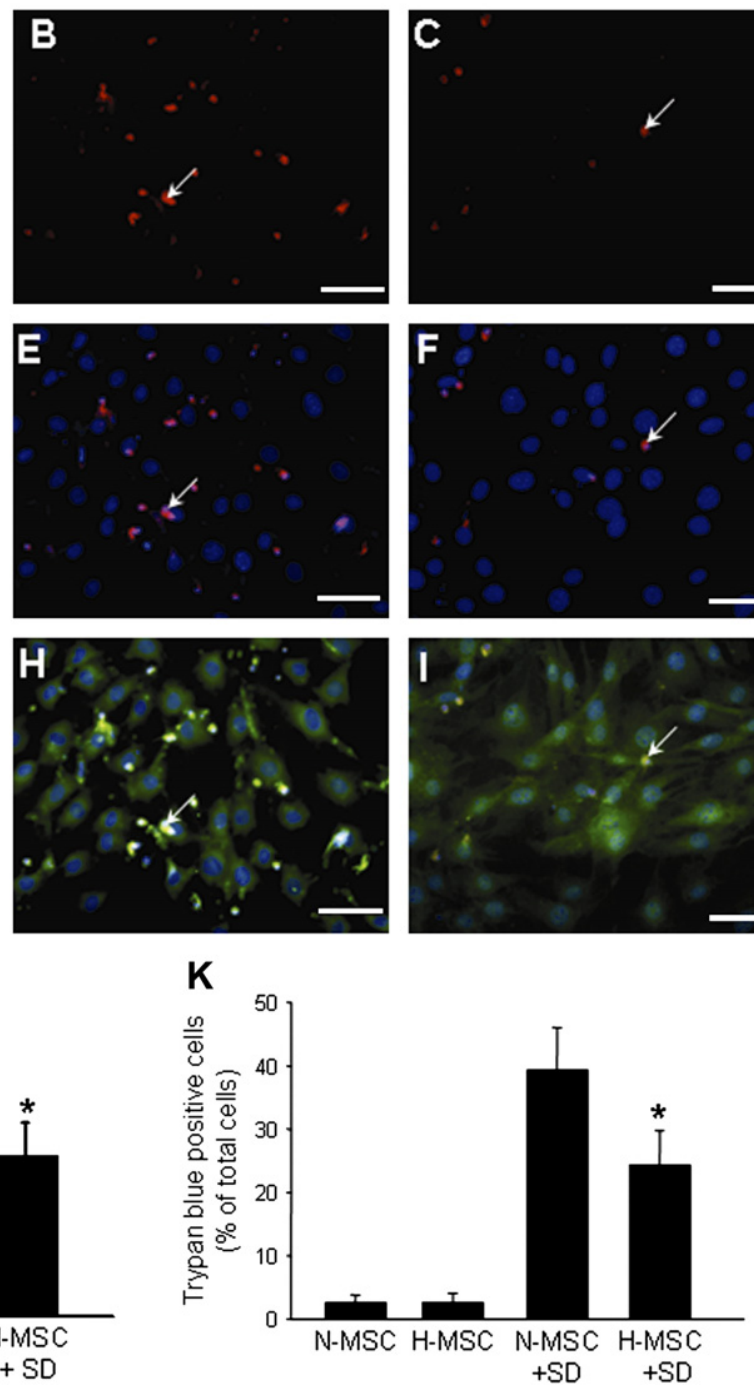

Figure 3. Effect of hypoxic preconditioning on cell death and cell survival in vitro. GFP MSC nuclei were prestained with Hoechst 33342 (b/ue); the double labeling of GFP and nuclear stain facilitated cell counting of MSCs. Cell apoptosis was identified by the antibody against the cleaved/activated caspase-3 (red). A-I, Activated caspase-3 (arrow) staining in control, N-MSCs and H-MSCs subjected to 24hour SD. Bar $=\mathbf{5 0} \mu \mathrm{m}$. J and K, Summarized data of caspase-3 activation for apoptosis $(\mathrm{J})$ and trypan blue staining for cell death (K). The ratio of cell apoptosis and cell death were significantly increased after SD, and hypoxic preconditioning significantly reduced apoptosis. $\mathbf{N}=4{ }^{*}{ }^{*} \boldsymbol{P}<.05$ compared with N-MSCs plus SD. GFP, Green fluorescent protein; $S D$, serum deprivation; for other abbreviations, see Figure 1. cells transplanted into ischemic myocardium were immunologically tolerant and feasible. ${ }^{24}$ Therefore, immunosuppression was not given in the present investigation and no rejection of implanted cells was noticed. Transplanted bone marrow MSCs are sensitive to ischemic and inflammatory microenvironment as is evidenced by stem cell death that often occurs soon after transplantation. ${ }^{6,8,25}$ This cell death may, in turn, increase the inflammatory response and be an additional burden to the ischemic heart, hindering functional recovery. Gene modification has been investigated to enhance stem cell survival and efficiency of cell transplantation therapy. For instance, overexpression of Akt can reduce apoptosis of MSCs and then enhance the heart functional improvement. ${ }^{26}$ Our previous study showed that transplantation of embryonic stem cells overexpressing Bcl-2 increased the survival and neuronal differentiation of transplanted cells, as well as functional recovery after cerebral ischemic stroke. ${ }^{27}$
Whether or not permanent gene modification has a longterm risk of tumorigenesis is not clear, ${ }^{28}$ which may limit its clinical utility. On the basis of this concern, increasing attention has been paid to short but comprehensive improvements of the quality of transplanted cells. The present study supports that HP may be explored as such a strategy in cell transplantation therapy for ischemic heart diseases.

Considering that up to $90 \%$ of grafted cells may die within the first few days of transplantation, the transient cytoprotective effect of HP should be sufficient to protect transplanted cells during the initial critical period after transplantation. Enhanced implanted cell survival can reduce the required number of transplanted cells, and fewer stem cells may actually differentiate better. Transplantation of MSC was performed 30 minutes after MI. In this case, some observed beneficial effects might result from myocardial rescue that prevented negative remodeling during the acute phase. Therapeutic 

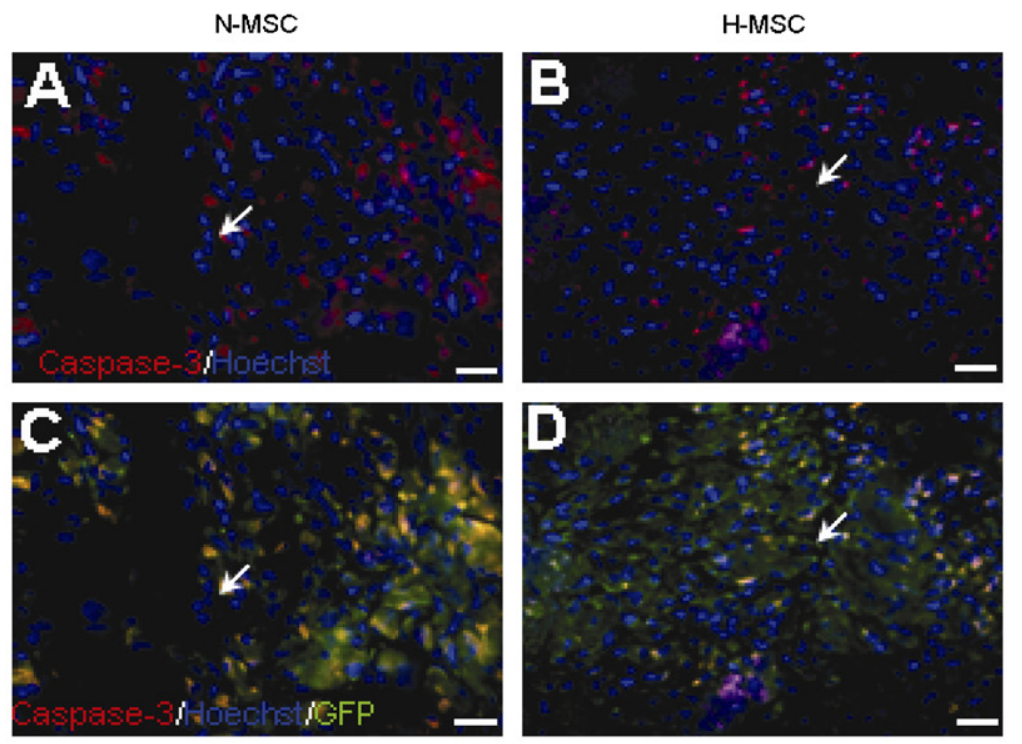

N-MSC
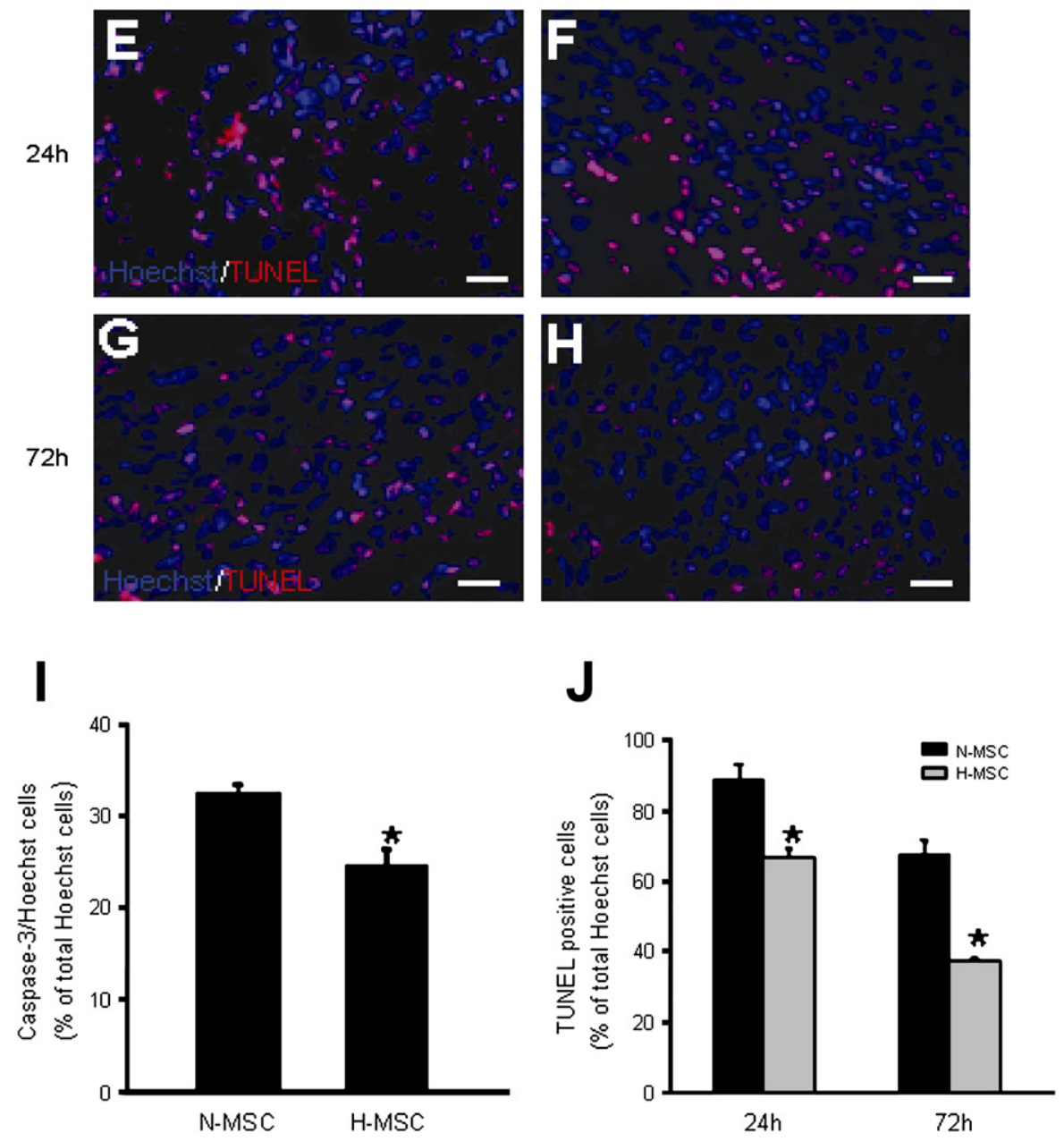

Figure 4. Effect of hypoxic preconditioning on implanted cell death 1 to 3 days after myocardial infarction. MSCs were labeled with Hoechst 33342 (blue) before transplantation. Cell apoptosis was identified with activated caspase-3, cell death was identified with TUNEL staining. A and B, Co-labeling of activated caspase-3 (red) and Hoechst (blue arrows) indicates apoptosis of graft cells in the ischemic heart 24 hours after myocardial infarction. C and D, Merged images of caspase-3/Hoechst/GFP that further confirmed the apoptosis of implanted cells. $E$ and $F$, Co-labeling of TUNEL (red) and Hoechst (blue) 24 hours after myocardial infarction in N-MSC and $\mathrm{H}$ MSC transplantation group, respectively. G and $\mathrm{H}$, Co-labeling of TUNEL and Hoechst 72 hours after myocardial infarction. Bar $=20 \mu \mathrm{m}$. I and J, Summarized data of caspase-3 activation for apoptosis (I) and TUNEL staining for cell death (J). Hypoxic preconditioning significantly reduced cell death and apoptosis of transplanted MSCs in the ischemic heart. ${ }^{*} P<.05$ compared with N-MSC. TUNEL, Terminal deoxynucleotidyl transferase biotin-dUPT nick end labeling. For other abbreviations, see Figure 1. 

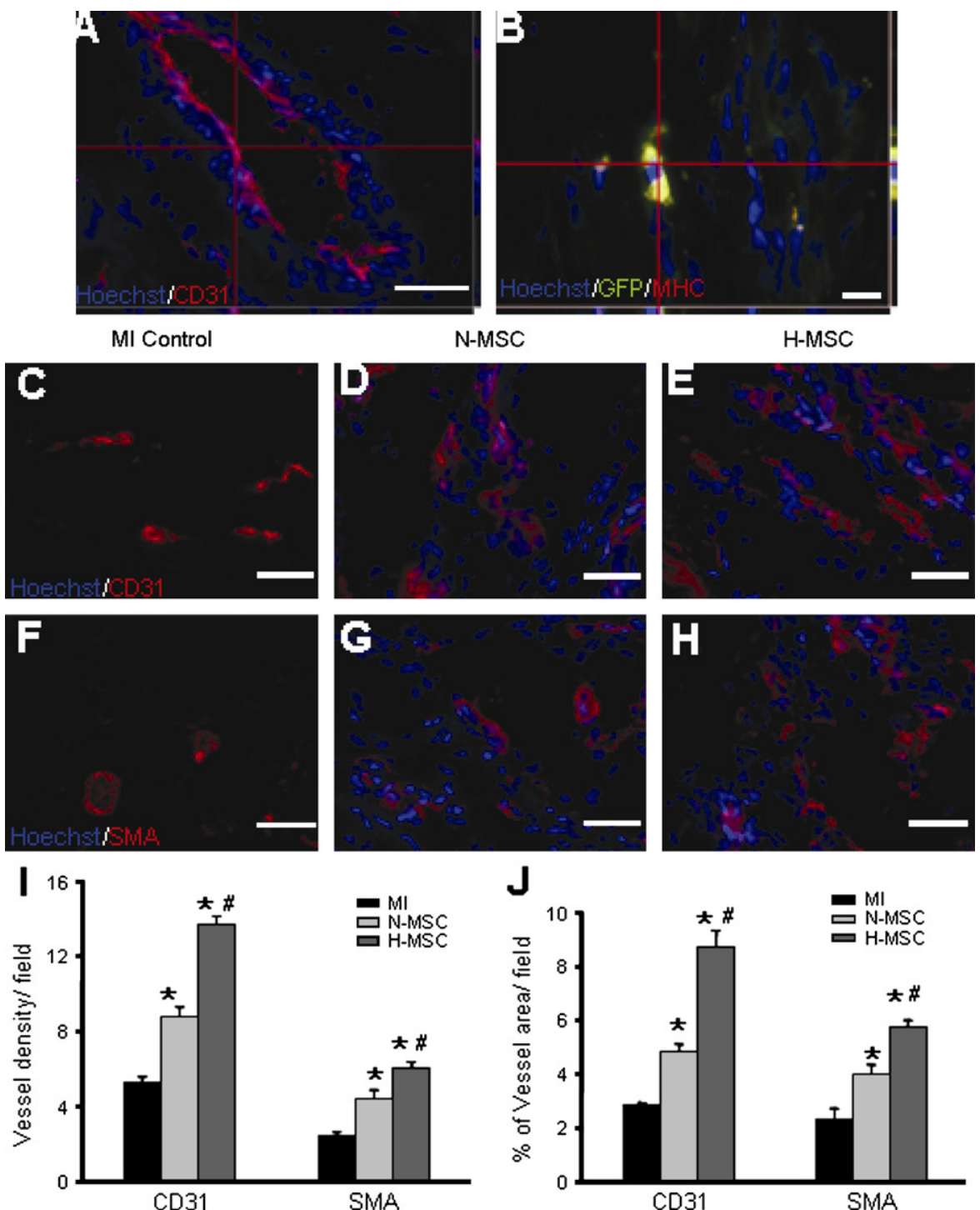

1

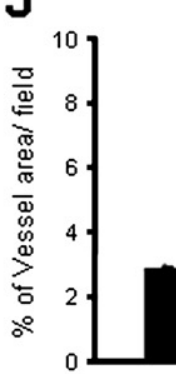

CD31

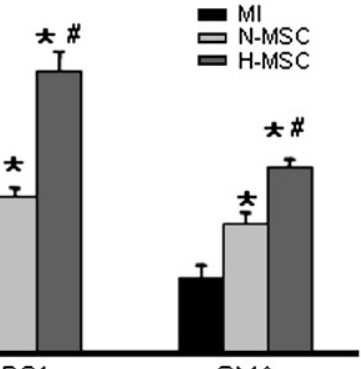

SMA

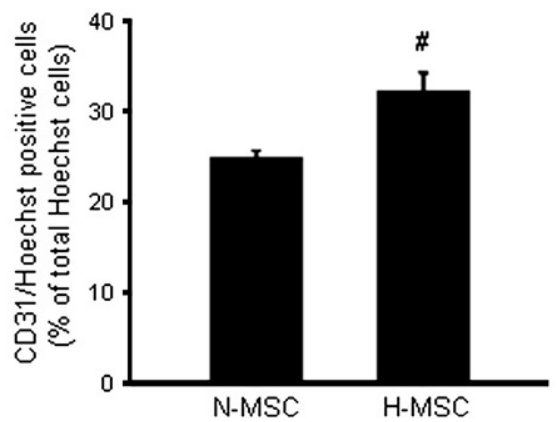

potential of transplantation of MSCs in the chronic phase long after MI remains to be examined. MSCs may provide host tissues and themselves with trophic support. MSCs can express cytokines and growth factors that play important roles in cell survival and angiogenesis, such as VEGF, basic
Figure 5. Differentiation of transplanted cells in ischemic heart 6 weeks after MI. Immunohistochemical staining of heart sections at transplanted sites after MI and transplantation. MSCs were labeled with Hoechst 33342 before transplantation, and vascular endothelial cells were stained with CD31, arteriole marker was stained with SMA and cardiomycyte marker was stained with myosin heavy chain. A, Three-dimensional image shows a vascular-like structure positively labeled with Hoechst (blue) and CD31 (red). Bar = $40 \mu \mathrm{m}$. B, Three-dimensional image of Hoechst/MHC and GFP (green), indicating some graft cells develop into cardiac-like myocytes. Bar $=10 \mu \mathrm{m}$. C-E, Merged images of Hoechst and CD31 (red) staining in three experimental groups; F-H, Merged images of Hoechst 33342 and SMA (red) staining in three experimental groups; increased numbers of CD31-positive vessels and SMA-positive arterioles were seen in the MSC transplantation groups. Bar = $40 \mu \mathrm{m}$. I-K, Summary of total vessel density and arteriole density (I), area per field $(\mathrm{J})$ and endothelial differentiation ratio (K) in three experimental groups. Angiogenic and/or vasculogenic activities were enhanced in the MSC transplantation rats; H-MSCs showed stronger angiogenic potency compared with $\mathbf{N}$-MSCs. $\mathbf{N}=6$. ${ }^{*} P<.05$ compared with MI group. ${ }^{\#} \boldsymbol{P}<.05$ compared with N-MSC group. MI, Myocardial infarction; SMA, smooth muscle actin. For other abbreviations, see Figure 1.

fibroblast growth factor, angiopoietin-1, and EPO. ${ }^{12,29}$ Our study demonstrates that MSCs express these factors, which are up-regulated by HP, in line with previous studies. ${ }^{21,30}$ These growth factors exert both autocrine and paracrine effects. HP augmented paracrine signaling and reduced 

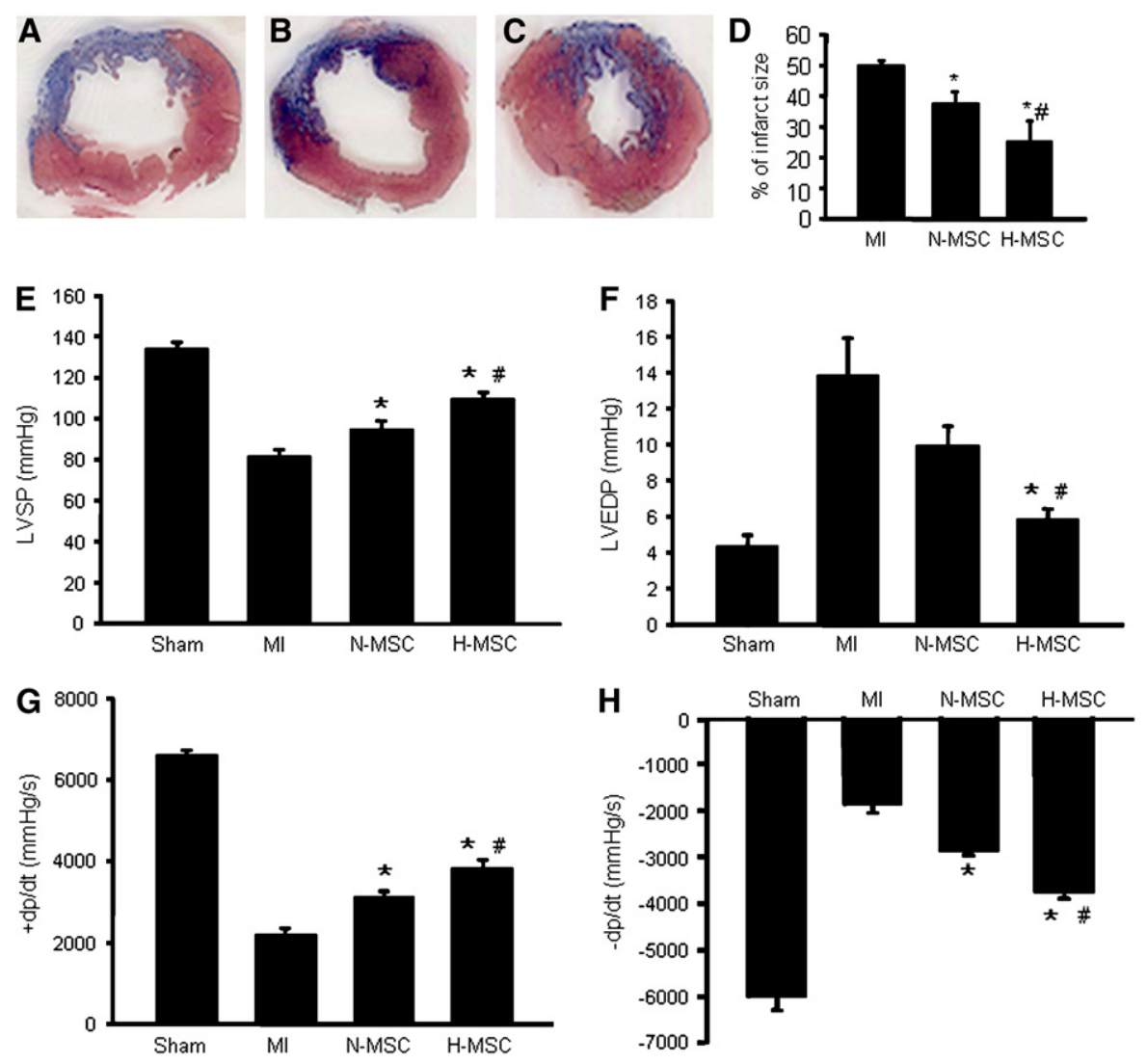

Figure 6. MSC transplantation reduced MI-induced infarct size and improved heart function. A-C, Masson trichrome staining reveals infarct and scar formation 6 weeks after MI. N-MSC transplantation inhibited the infarct size compared with the MI-only group (B). The smallest infarct size was seen in the H-MSC transplantation group (C). $D$, Summary of infarct size in three experimental groups. E, LVSP decreased significantly 6 weeks after MI compared with sham group. While MSC transplantation improved LVSP, HMSC transplantation showed stronger effect on LVSP recovery. $F$, LVEDP increased markedly 6 weeks after Ml; N-MSC transplantation showed no significant effect while H-MSC transplantation enhanced LVEDP to a near normal level. $G$ and $H$, Left ventricular $+\mathrm{dp} / \mathrm{dt}(\mathrm{G})$ and $-\mathrm{dp} / \mathrm{dt}(\mathrm{H})$ decreased 6 weeks after MI; both transplantation groups increased $\pm \mathrm{dp} / \mathrm{dt}$, especially in the H-MSC transplantation group. $\mathbf{N}=$ 6. ${ }^{*} \boldsymbol{P}<.05$ as compared with the MIonly group. ${ }^{\#} P<.05$ as compared with N-MSC transplantation group. MSC, Mesenchymal stem cell $(N$, normoxic; $H$, hypoxic); $M I$, myocardial infarction; LVSP, left ventricular systolic pressure; LVEDP, left ventricular end-diastolic pressure; $d p / d t$, rate of pressure rise. cardiomyocytes apoptosis. $^{21,30}$ Several transcription factors may be involved in the response to hypoxia, such as activated protein-1, HIF- $1 \alpha$, and nuclear factor- $\kappa \mathrm{B} .{ }^{31}$ Activation of some growth factor pathways can activate nuclear factor $-\kappa \mathrm{B}$ and increase the survival genes such as Bcl-2 and Bcl-xL. ${ }^{32}$ Consistent with these anti-apoptotic effects, caspase- 3 activation is suppressed in H-MSCs. It is likely that a combination of these multiple mechanisms is responsible for the increased viability of H-MSCs.

In this study, MI-induced animal death mostly occurred 3 days after surgery, which was not affected by H-MSC transplantation. The lack of the effect on animal mortality suggests that although the hypoxic preconditioned MSCs survives better in the ischemic heart, the increased cell survival is not sufficient to antagonize the initial overwhelming insults and functional failure in the acute phase of MI. Moreover, the H-MSC-promoted cell survival and angiogenesis are expected to primarily benefit long-term tissue repair and function recovery many days after $\mathrm{MI}$, as shown in this investigation.

An earlier study showed that ex vivo HP up-regulated the synthesis of VEGF messenger RNA and stimulated endothe- lial differentiation of bone marrow stem cells, which together contributed to improved angiogenesis in the ischemic hind limb after transplantation. ${ }^{33}$ Our finding that HP increased vessel density, area, and the endothelial cell differentiation ratio is consistent with this previous study. The higher vessel density in H-MSC transplantation group is attributable to both enhanced paracrine effect and increased endothelial cell differentiation. Several groups have reported that MSCs can differentiate into cardiomyocytes ${ }^{9,34}$; other studies question the possibility that MSCs might fuse with host cells after transplantation into the ischemic heart. ${ }^{6,7}$ Our study identified that, 6 weeks after transplantation, a few MSCs showed characteristic myosin protein expression. Whether transplanted MSCs may undergo cardiac myocyte differentiation is under debate. Moreover, possible fusion and uptake of Hoechst dye by endogenous cells might also affect the endothelial cell counts. In any event, transplanted MSCs helped to repair infarcted myocardium, disregarding the rare events of myogenesis or cell fusion. ${ }^{35}$

In conclusion, our study demonstrates that transplantation of HP-treated MSCs shows better therapeutic effects in the ischemic heart. The functional benefit of H-MSC 
transplantation might be explained by several possibilities: (1) HP enhances the autocrine and paracrine signaling of MSCs, which reduces apoptosis of transplanted cells and endogenous cardiomyocytes; (2) the increased survival of H-MSCs provides better and longer trophic support for the reparative process; (3) the increase in survival of engrafted cells contributes to increased angiogenesis. These factors collectively promote tissue repair and may provide a simple but effective new strategy for clinical MSC transplantation therapy. Of note, although HP of MSCs may show the above benefits, transplantation of H-MSCs did not improve short-term animal survival 3 days after MI; its effect on long-term mortality remains to be tested.

\section{References}

1. Braunwald E, Bristow MR. Congestive heart failure: fifty years of progress. Circulation. 2000;102(20 Suppl. 4):IV14-23.

2. Pittenger MF, Mackay AM, Beck SC, Jaiswal RK, Douglas R, Mosca JD, et al. Multilineage potential of adult human mesenchymal stem cells. Science. 1999;284:143-7.

3. Orlic D, Kajstura J, Chimenti S, Jakoniuk I, Anderson SM, Li B, et al. Bone marrow cells regenerate infarcted myocardium. Nature. 2001; 410:701-5.

4. Wollert KC, Meyer GP, Lotz J, Ringes-Lichtenberg S, Lippolt P, Breidenbach $\mathrm{C}$, et al. Intracoronary autologous bone-marrow cell transfer after myocardial infarction: the BOOST randomised controlled clinical trial. Lancet. 2004;364:141-8.

5. Tomita S, Li RK, Weisel RD, Mickle DA, Kim EJ, Sakai T, et al. Autologous transplantation of bone marrow cells improves damaged heart function. Circulation. 1999;100(19 Suppl):II247-56.

6. Zhang M, Methot D, Poppa V, Fujio Y, Walsh K, Murry CE. Cardiomyocyte grafting for cardiac repair: graft cell death and anti-death strategies. J Mol Cell Cardiol. 2001;33:907-21.

7. Nygren JM, Jovinge S, Breitbach M, Sawen P, Roll W, Hescheler J, et al. Bone marrow-derived hematopoietic cells generate cardiomyocytes at a low frequency through cell fusion, but not transdifferentiation. Nat Med. 2004;10:494-501.

8. Reinecke H, Murry CE. Cell grafting for cardiac repair. Methods Mol Biol. 2003;219:97-112.

9. Toma C, Pittenger MF, Cahill KS, Byrne BJ, Kessler PD. Human mesenchymal stem cells differentiate to a cardiomyocyte phenotype in the adult murine heart. Circulation. 2002;105:93-8.

10. Silva GV, Litovsky S, Assad JA, Sousa AL, Martin BJ, Vela D, et al. Mesenchymal stem cells differentiate into an endothelial phenotype, enhance vascular density, and improve heart function in a canine chronic ischemia model. Circulation. 2005;111:150-6.

11. Isner JM. Angiogenesis: a "breakthrough" technology in cardiovascular medicine. J Invasive Cardiol. 2000;12(Suppl A):14A-17A.

12. Hamano K, Li TS, Kobayashi T, Kobayashi S, Matsuzaki M, Esato K. Angiogenesis induced by the implantation of self-bone marrow cells: a new material for therapeutic angiogenesis. Cell Transplant. 2000;9: 439-43.

13. Liu J, Narasimhan P, Yu F, Chan PH. Neuroprotection by hypoxic preconditioning involves oxidative stress-mediated expression of hypoxiainducible factor and erythropoietin. Stroke. 2005;36:1264-9.

14. Uchiyama T, Engelman RM, Maulik N, Das DK. Role of Akt signaling in mitochondrial survival pathway triggered by hypoxic preconditioning. Circulation. 2004;109:3042-9.

15. Sasaki H, Fukuda S, Otani H, Zhu L, Yamaura G, Engelman RM, et al. Hypoxic preconditioning triggers myocardial angiogenesis: a novel approach to enhance contractile functional reserve in rat with myocardial infarction. J Mol Cell Cardiol. 2002;34:335-48.
16. Hedrick MY, Yu SP, Wei L. Preconditioning with sublethal hypoxia induces protection in neural-derived embryonic stem cells in vitro. Exp Neurol. 2005;193:247-8.

17. Campagnoli C, Roberts IA, Kumar S, Bennett PR, Bellantuono I, Fisk NM. Identification of mesenchymal stem/progenitor cells in human first-trimester fetal blood, liver, and bone marrow. Blood. 2001;98: 2396-402.

18. Abdalah R, Wei L, Francis K, Yu SP. Valinomycin-induced apoptosis in Chinese hamster ovary cells. Neurosci Lett. 2006;405:68-73.

19. Min JY, Sandmann S, Meissner A, Unger T, Simon R. Differential effects of mibefradil, verapamil, and amlodipine on myocardial function and intracellular $\mathrm{Ca}(2+)$ handling in rats with chronic myocardial infarction. J Pharmacol Exp Ther. 1999;291:1038-44.

20. Leenen FH, Huang BS, Yu H, Yuan B. Brain "ouabain" mediates sympathetic hyperactivity in congestive heart failure. Circ Res. 1995;77: 993-1000

21. Uemura R, Xu M, Ahmad N, Ashraf M. Bone marrow stem cells prevent left ventricular remodeling of ischemic heart through paracrine signaling. Circ Res. 2006;98:1414-21.

22. Di Nicola M, Carlo-Stella C, Magni M, Milanesi M, Longoni PD, Matteucci $\mathrm{P}$, et al. Human bone marrow stromal cells suppress T-lymphocyte proliferation induced by cellular or nonspecific mitogenic stimuli. Blood. 2002;99:3838-43.

23. Saito T, Kuang JQ, Bittira B, Al-Khaldi A, Chiu RC. Xenotransplant cardiac chimera: immune tolerance of adult stem cells. Ann Thorac Surg. 2002;74:19-24; discussion 24.

24. MacDonald DJ, Luo J, Saito T, Duong M, Bernier PL, Chiu RC, et al. Persistence of marrow stromal cells implanted into acutely infarcted myocardium: observations in a xenotransplant model. J Thorac Cardiovasc Surg. 2005;130:1114-21.

25. Reinecke H, Murry CE. Transmural replacement of myocardium after skeletal myoblast grafting into the heart. Too much of a good thing? Cardiovasc Pathol. 2000;9:337-44.

26. Mangi AA, Noiseux N, Kong D, He H, Rezvani M, Ingwall JS, et al. Mesenchymal stem cells modified with Akt prevent remodeling and restore performance of infarcted hearts. Nat Med. 2003;9:1195-201.

27. Wei L, Cui L, Snider BJ, Rivkin M, Yu SS, Lee CS, et al. Transplantation of embryonic stem cells overexpressing Bcl-2 promotes functional recovery after transient cerebral ischemia. Neurobiol Dis. 2005;19:183-93.

28. Meuillet EJ, Mahadevan D, Vankayalapati H, Berggren M, Williams R, Coon A, et al. Specific inhibition of the Akt1 pleckstrin homology domain by D-3-deoxy-phosphatidyl-myo-inositol analogues. Mol Cancer Ther. 2003;2:389-99.

29. Kinnaird T, Stabile E, Burnett MS, Lee CW, Barr S, Fuchs S, et al. Marrow-derived stromal cells express genes encoding a broad spectrum of arteriogenic cytokines and promote in vitro and in vivo arteriogenesis through paracrine mechanisms. Circ Res. 2004;94:678-85.

30. Takahashi M, Li TS, Suzuki R, Kobayashi T, Ito H, Ikeda Y, et al. Cytokines produced by bone marrow cells can contribute to functional improvement of the infarcted heart by protecting cardiomyocytes from ischemic injury. Am J Physiol Heart Circ Physiol. 2006;291:H886-93.

31. Faller DV. Endothelial cell responses to hypoxic stress. Clin Exp Pharmacol Physiol. 1999;26:74-84.

32. Digicaylioglu M, Lipton SA. Erythropoietin-mediated neuroprotection involves cross-talk between Jak2 and NF-kappaB signalling cascades. Nature. 2001;412:641-7.

33. Li TS, Hamano K, Suzuki K, Ito H, Zempo N, Matsuzaki M. Improved angiogenic potency by implantation of ex vivo hypoxia prestimulated bone marrow cells in rats. Am J Physiol Heart Circ Physiol. 2002; 283:H468-73.

34. Kajstura J, Rota M, Whang B, Cascapera S, Hosoda T, Bearzi C, et al. Bone marrow cells differentiate in cardiac cell lineages after infarction independently of cell fusion. Circ Res. 2005;96:127-37.

35. Noiseux N, Gnecchi M, Lopez-Ilasaca M, Zhang L, Solomon SD, Deb A, et al. Mesenchymal stem cells overexpressing Akt dramatically repair infarcted myocardium and improve cardiac function despite infrequent cellular fusion or differentiation. Mol Ther. 2006;14:840-50. 